\title{
Nano-Resolved Current-Induced Insulator-Metal Transition in the Mott Insulator $\mathrm{Ca}_{2} \mathrm{RuO}_{4}$
}

\author{
Jiawei Zhang, ${ }^{1, *}$ Alexander S. McLeod, ${ }^{2, *}$ Qiang Han, ${ }^{2, *}$ Xinzhong Chen, ${ }^{1}$ Hans A. Bechtel, ${ }^{3}$ Ziheng Yao, ${ }^{1}$ \\ S. N. Gilbert Corder, ${ }^{1}$ Thomas Ciavatti, ${ }^{1}$ Tiger H. Tao, ${ }^{4}$ Meigan Aronson, ${ }^{5}$ G. L. Carr, ${ }^{6}$ Michael C. Martin, ${ }^{3}$ Chanchal Sow, ${ }^{7}$ \\ Shingo Yonezawa, ${ }^{7}$ Fumihiko Nakamura, ${ }^{8}$ Ichiro Terasaki, ${ }^{9}$ D. N. Basov, ${ }^{2}$ \\ Andrew J. Millis, ${ }^{2,10, \uparrow}$ Yoshiteru Maeno, ${ }^{7, \$}$ and Mengkun Liu ${ }^{1, \Phi}$ \\ ${ }^{1}$ Department of Physics, Stony Brook University, Stony Brook, New York 11794, USA \\ ${ }^{2}$ Department of Physics, Columbia University, New York, New York 10027, USA \\ ${ }^{3}$ Advanced Light Source, Lawrence Berkeley National Laboratory, Berkeley, California 94720, USA \\ ${ }^{4}$ State Key Laboratory of Transducer Technology, Shanghai Institute of Microsystem and Information \\ Technology, Chinese Academy of Sciences, Shanghai 200050, China \\ ${ }^{5}$ Department of Physics, Texas A\&M University, College Station, Texas 77843, USA \\ ${ }^{6}$ NSLS-II Photon Sciences, Brookhaven National Laboratory, Upton, New York 11973, USA \\ ${ }^{7}$ Department of Physics, Kyoto University, Kyoto 606-8502, Japan \\ ${ }^{8}$ Department of Education and Creation Engineering, Kurume Institute of Technology, \\ Fukuoka 830-0052, Japan \\ ${ }^{9}$ Department of Physics, Nagoya University, Nagoya 464-8602, Japan \\ ${ }^{10}$ Center for Computational Quantum Physics, The Flatiron Institute, $1625^{\text {th }}$ Avenue, \\ New York, New York 10010, USA
}

(Received 29 July 2018; revised manuscript received 16 December 2018; published 15 February 2019)

The Mott insulator $\mathrm{Ca}_{2} \mathrm{RuO}_{4}$ is the subject of much recent attention following reports of emergent nonequilibrium steady states driven by applied electric fields or currents. In this paper, we carry out infrared nano-imaging and optical-microscopy measurements on bulk single crystal $\mathrm{Ca}_{2} \mathrm{RuO}_{4}$ under conditions of steady current flow to obtain insight into the current-driven insulator-tometal transition. We observe macroscopic growth of the current-induced metallic phase, with nucleation regions for metal and insulator phases determined by the polarity of the current flow. A remarkable metal-insulator-metal microstripe pattern is observed at the phase front separating metal and insulator phases. The microstripes have orientations tied uniquely to the crystallographic axes, implying a strong coupling of the electronic transition to lattice degrees of freedom. Theoretical modeling further illustrates the importance of the current density and confirms a submicron-thick surface metallic layer at the phase front of the bulk metallic phase. Our work confirms that the electrically induced metallic phase is nonfilamentary and is not driven by Joule heating, revealing remarkable new characteristics of electrically induced insulator-metal transitions occurring in functional correlated oxides.

\section{INTRODUCTION}

Electric control of nonthermal phase transitions in strongly correlated electron materials (SCEMs) is a central theme of modern-day condensed-matter research [1-9]. While many

\footnotetext{
*These authors contributed equally to the present work.

Corresponding authors. ajm2010@columbia.edu

\#maeno@scphys.kyoto-u.ac.jp

"mengkun.liu@stonybrook.edu
}

Published by the American Physical Society under the terms of the Creative Commons Attribution 4.0 International license. Further distribution of this work must maintain attribution to the author(s) and the published article's title, journal citation, and DOI. observations of current- or field-driven transitions are reported, interpretation in terms of true nonequilibrium phases is complicated by the possibilities of Joule heating (which could imply that the current simply heats the system into the higher-temperature phase) and microscopic phase separation. This possibility is especially true for SCEMs, since the competition between many degrees of freedom including orbital, lattice, and magnetic ordering can yield the coexistence of multiple phases in the same crystal, triggered by minute perturbations of the electronic and lattice subsystems. Field-induced filament growth and temperature-induced phase percolation at microscopic scales are extensively reported in $3 \mathrm{~d}$ transition-metal oxides, including vanadates, manganites, and cuprates [10-17]. Therefore, direct insight into the spatial structure and thermal aspects of quantum phase transitions is urgently needed. 
In this paper, we study the paradigmatic $4 \mathrm{~d}$ transitionmetal oxide $\mathrm{Ca}_{2} \mathrm{RuO}_{4}$. At thermal equilibrium, $\mathrm{Ca}_{2} \mathrm{RuO}_{4}$ is a Mott insulator at room temperature and a "bad metal" at high temperatures [18]. Both heating above $T_{c}=357 \mathrm{~K}$ [19] and applying hydrostatic pressure above $0.5 \mathrm{GPa}$ [20-22] can induce the insulator-to-metal transition (IMT). It was recently found that an astonishingly small electric field of $40 \mathrm{~V} / \mathrm{cm}$ can also induce the IMT, with experimental evidence excluding the major role of Joule heating $[23,24]$. Furthermore, x-ray diffraction measurements reveal a possible intermediate electronic and lattice state preceding the full metallic phase, maintained by dc current [25]. These current-induced states in $\mathrm{Ca}_{2} \mathrm{RuO}_{4}$ can persist to low temperatures, where strong diamagnetism was discovered at a current density of merely $10 \mathrm{~A} / \mathrm{cm}^{2}$ [2]. Therefore, a mounting body of evidence suggests the importance of current-induced nonequilibrium phenomena in $\mathrm{Ca}_{2} \mathrm{RuO}_{4}$. However, the microscopic spatial structure of the metallic phase and the transition itself have heretofore not been clarified.

We use IR nanospectroscopy and nano-imaging techniques based on scattering-type scanning near-field optical microscopy (s-SNOM) [26,27]. The experiments are performed at room temperature and ambient pressure, under conditions of a controlled constant current flow. The IR nanospectroscopy covers a wide frequency range from 400 to $2000 \mathrm{~cm}^{-1}(25-5 \mu \mathrm{m})$ capturing both the phonon and electronic optical response with $20 \mathrm{~nm}$ spatial resolution. In addition, $\mathrm{CO}_{2}$ laser-based IR nano-imaging at approximately $900 \mathrm{~cm}^{-1}$ (about $11 \mu \mathrm{m}$ ) with the same spatial resolution is used to investigate the current-induced insulator-metal phase boundary across different stages of the phase transition [27]. In agreement with previous results, we find that at low current densities the sample is entirely insulating, while at high current densities the sample is entirely metallic. At intermediate currents, we find new effects: As the current is increased above a critical value, an apparently uniform (nonfilamentary) metallic phase grows out of the negative electrode, covering a progressively greater portion of the sample until it is entirely transformed. Reversing the polarity of the electrodes reverses the electrode from which the metallic phase emerges and the direction of phase propagation. This polarity dependence rules out Joule heating as a fundamental transition mechanism. Moreover, IR s-SNOM imaging of the insulatormetal phase boundary sustained by the electric current reveals exotic stripelike patterns of phase coexistence which, to the best of our knowledge, have not been observed in previous studies of the IMT in single crystals of correlated electron oxides [28-32]. We formulate a minimal theory accounting for the current density-sustained IMT and the influence of long-range strain which identifies the spontaneous stripe pattern as the hallmark of strong coupling between the nonequilibrium Mott transition and induced structural distortions of the $\mathrm{Ru}-\mathrm{O}_{6}$ octahedra.

\section{CURRENT-INDUCED INSULATOR-TO-METAL TRANSITION}

\section{A. Macroscopic imaging}

Our experiments examine $\mathrm{Ca}_{2} \mathrm{RuO}_{4}$ single crystals, with typical dimension of $\sim 1 \mathrm{~mm} \times \sim 1 \mathrm{~mm} \times \sim 200 \mu \mathrm{m}$ (thickness). Current is introduced to the sample via needlelike electrodes with a width of approximately $40 \mu \mathrm{m}$ deposited on opposite edges of the crystal, enabling two terminal $I-V$ characteristics to be obtained simultaneously with the acquisition of optical micrographs and s-SNOM imaging across the IMT. Schematics of the experimental setup are shown in Fig. S1 [27] of Supplemental Material. The main panel in Fig. 1(a) presents the two-terminal $I-V$ characteristic. Simultaneously acquired optical micrographs obtained under visible light illumination are shown as insets. As the current is increased from zero, the sample remains insulating (low $I-V$ ratio, region 1) up to a critical voltage of about $5 \mathrm{~V}$ (electric field $E \sim 50 \mathrm{~V} / \mathrm{cm}$ for our sample dimension). The corresponding optical micrograph shows that the sample is insulating (visibly bright) at zero voltage. This phase is labeled as the $S$ phase to be consistent with the previously reported $S$-Pbca lattice structure of the insulating phase [33,34]. At slightly above $5 \mathrm{~V}$, the current discontinuously jumps to a higher value. At the same time, a visibly dark region (identified as the metallic $L$-Pbca state and denoted here as $L$ ) nucleates from the negative electrode and expands with an increasing current. The boundary of the $L$ phase is seen to have a convex arclike shape surrounding the effectively pointlike electrode, suggesting the IMT occurs only where the current density exceeds a critical value, thus determining the location of the insulator-metal boundary. As the current increases, the area of the metallic (dark) region increases. The $I-V$ curve (curve 2) correspondingly displays a negative differential resistance which correlates with the optically identified volume fraction of the metal. The sample is completely transformed at sufficiently high currents.

Remarkably, as demonstrated in Figs. 1(b) and 1(c), the $L$ phase consistently emerges from the negative electrodes for all samples we test. Reversing the polarity of the applied voltage also reverses the electrode from which the $L$ phase emerges. These observations strongly weaken the importance of Joule heating, which should be a scalar quantity independent of the direction of the current. We also exclude a bulk Peltier effect as the dominant driving mechanism. The Peltier effect in $\mathrm{Ca}_{2} \mathrm{RuO}_{4}$ leads to a temperature gradient across the two electrodes, which might, in principle, drive the interface towards the positive electrode via thermoelectric heating. This scenario implies that the nucleated $L$ state has already reached $T_{c}$ and the movement of the phase boundary is purely due to local heating of the sample. However, as shown in Fig. S6 [27], we find that further heating of the entire sample within $18 \mathrm{~K}$ does not move the phase boundary. This observation counterindicates 


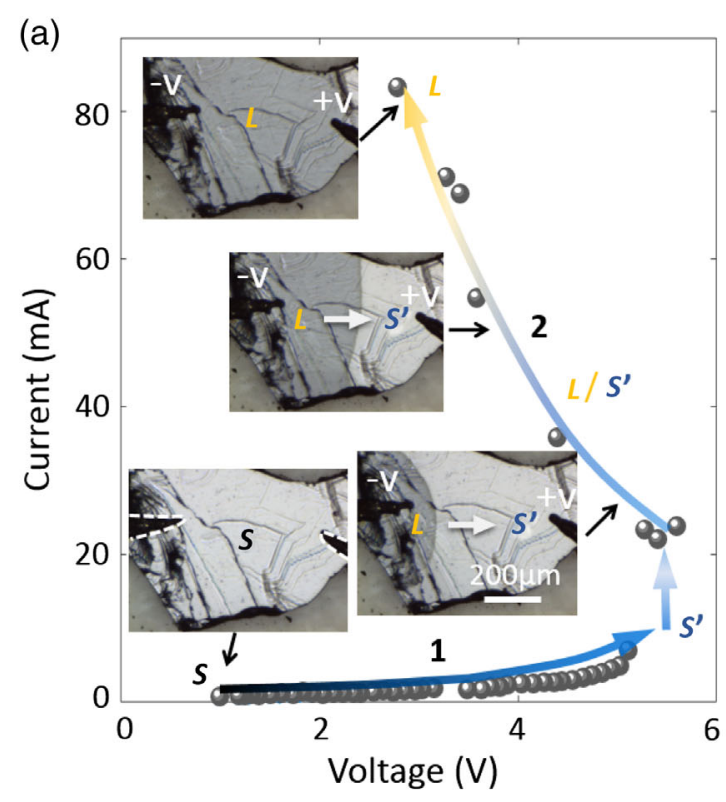

(b)

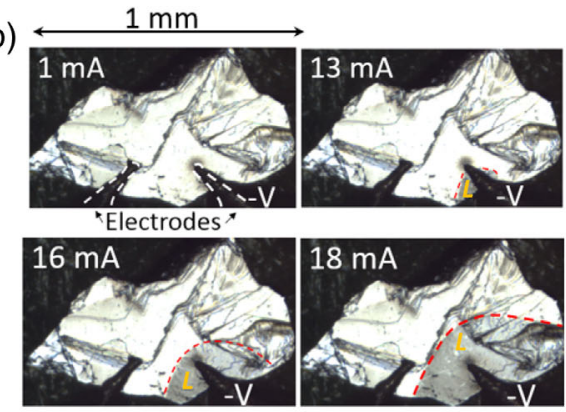

(c)

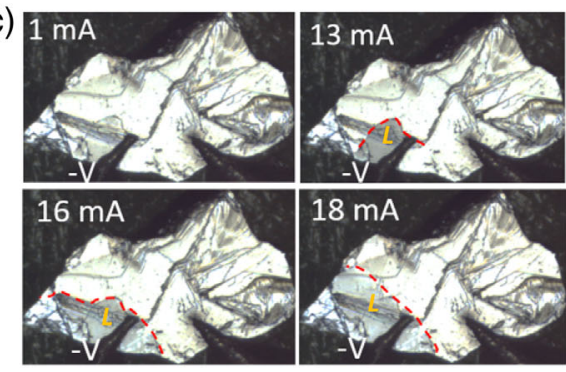

FIG. 1. de transport characterization and optical photographs of a $\mathrm{Ca}_{2} \mathrm{RuO}_{4}$ bulk single crystal at different stages of the IMT. (a) A dc $I-V$ curve with optical images taken by a CCD camera in the visible range. The insets show the emergence and expansion of the $L$ phase (dark region) at each stage of the phase transition. The white dashed line in the bottom inset outlines the silver paint electrodes on the sample surface. (b) and (c) show the switching of the $L$ phase from the right to left electrode via reversing the polarity of the two electrodes (outlined by the white dashed lines).

a thermoelectric mechanism and supports the scenario of an intrinsic electric-current-induced IMT in which bulk Joule or thermoelectric heating plays at most a minor role.

The microscopic origin of the polarity-dependent switching most likely arises from the strong electron-hole asymmetry of the many-body density of states of frontier (the $t_{2 g}$ symmetry $\mathrm{Ru} d$ ) orbitals. At room temperature in equilibrium, the gap in insulating $\mathrm{Ca}_{2} \mathrm{RuO}_{4}$ is formed between an $x y$-orbital-derived valence band and $x z / y z-$ orbital-derived upper-Hubbard bands. The resulting manybody density of states is highly asymmetric about the Fermi level, with the filled bands exhibiting a large peak at the band edge and the empty states having a smoother spectral structure [35]. During the IMT, there is a considerable redistribution of electrons from doubly occupied $x y$ (and singly occupied $x z / y z$ ) orbitals in the insulating phase to approximately equal the occupancy of each orbital in the metallic phase [36-38]. Therefore, under small changes in the electrochemical potential, the strong electron-hole asymmetry yields much more pronounced hole doping compared with electron doping in the metallic $L$ phase. This vast asymmetry in the electrostatic susceptibility of $\mathrm{Ca}_{2} \mathrm{RuO}_{4}$ may explain the initial nucleation of the $L$ phase at negative electrodes and may imply a current-dependent force across a domain wall.

\section{B. Nanoscale imaging and spectroscopy}

We collect broadband nano-IR spectra at different current-controlled stages of the IMT with approximately
$20 \mathrm{~nm}$ spatial resolution [Fig. 2(a)]. At $0 \mathrm{~V}$, the nano-IR spectrum reveals a peak at approximately $603 \mathrm{~cm}^{-1}$ with an apparent dip at about $680 \mathrm{~cm}^{-1}$ (gray curve, $S$ state). We identify this peak as the previously reported transverse optical in-plane Ru-O stretching mode of $\mathrm{Ca}_{2} \mathrm{RuO}_{4}$ [39-41]. The amplitude of this phonon response is continuously suppressed with an increasing electric current, and its peak position is weakly blueshifted (approximately $8 \mathrm{~cm}^{-1}$ ), which we take as signifiers of an intermediate state labeled as the $S^{\prime}$ state (blue curve; see also Fig. S3 [27]). The nano-IR spectrum collected in the $L$ phase (golden curve) shows a totally different behavior: The infrared signal presents an approximately fourfold increase over the entire spectrum range without the phonon peak at $603 \mathrm{~cm}^{-1}$. This increase, in combination with the visible brightness reduction in the metallic $L$ phase, suggests that the $L$ (metallic) phase is characterized by a large spectral weight transfer from higher energies (visible light frequencies) to lower energies (IR and dc) consistent with the onset of a Drude response. While the electromagnetic response of inhomogeneous mixtures is complicated, the absolute normalization and spatial resolution of our nano-IR spectroscopy shows that the IR signal of the $S^{\prime}$ state above $700 \mathrm{~cm}^{-1}$ is comparable to $S$ but much lower than $L$, ruling out the possibility that the $S^{\prime}$ state comprises any mixture of $L$ and $S$ phases. We attribute $S^{\prime}$ to a nonequilibrium intermediate state emerging under the electric current flow, distinct from $S$ and $L$ phases, in agreement with other experimental observations in $\mathrm{Ca}_{2} \mathrm{RuO}_{4}$ under similar steady-state current conditions [23,25]. 


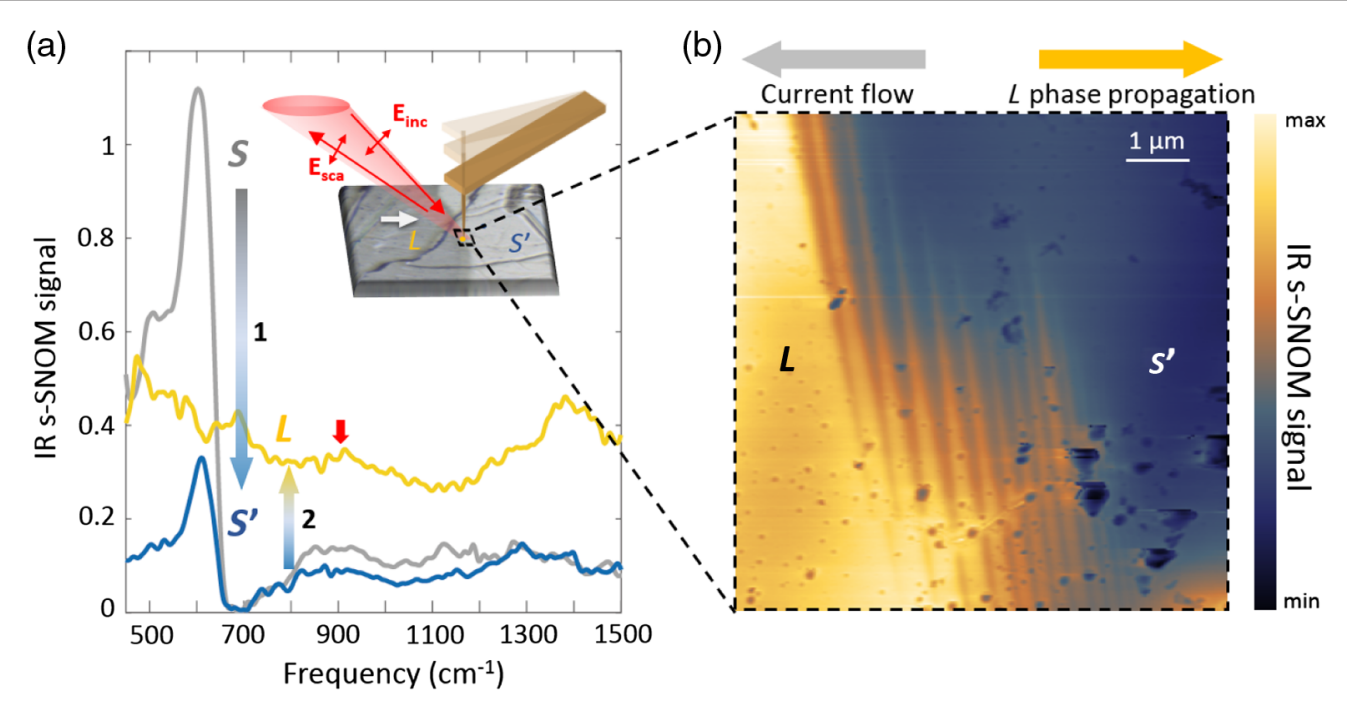

FIG. 2. (a) IR nano spectra of $S, S^{\prime}$, and $L$ states. The spectrum of $S$ (gray) is taken without a current, while the spectra of $S^{\prime}$ (blue) and $L$ (golden) are taken at two different regions several microns apart across the $L-S^{\prime}$ phase boundary under the same current. The spectra are normalized to that of a thick gold film. Inset: Schematic of the nanospectroscopy setup and the optical photo of the $L-S^{\prime}$ PB. (b) IR s-SNOM imaging (second harmonic) of the $L-S^{\prime}$ boundary stripes at the phase boundary at a frequency of $900 \mathrm{~cm}^{-1} \mathrm{marked} \mathrm{by} \mathrm{a}$ red arrow in (a).

We next report in Fig. 2(b) IR nano-imaging of the $L-S^{\prime}$ phase boundary $(\mathrm{PB})$ which is observed at intermediate currents. The probe laser at approximately $900 \mathrm{~cm}^{-1}$ (11 $\mu \mathrm{m}$ wavelength, or about $110 \mathrm{meV}$ ) is chosen to avoid energetic overlap with the transverse optical phonon response and to probe the genuine Drude response of the charge carriers in metallic $\mathrm{Ca}_{2} \mathrm{RuO}_{4}$. The higher IR response (more metallic) is rendered golden in false color, whereas dark blue represents a lower IR response characteristic of insulating regions. A striking pattern of alternating
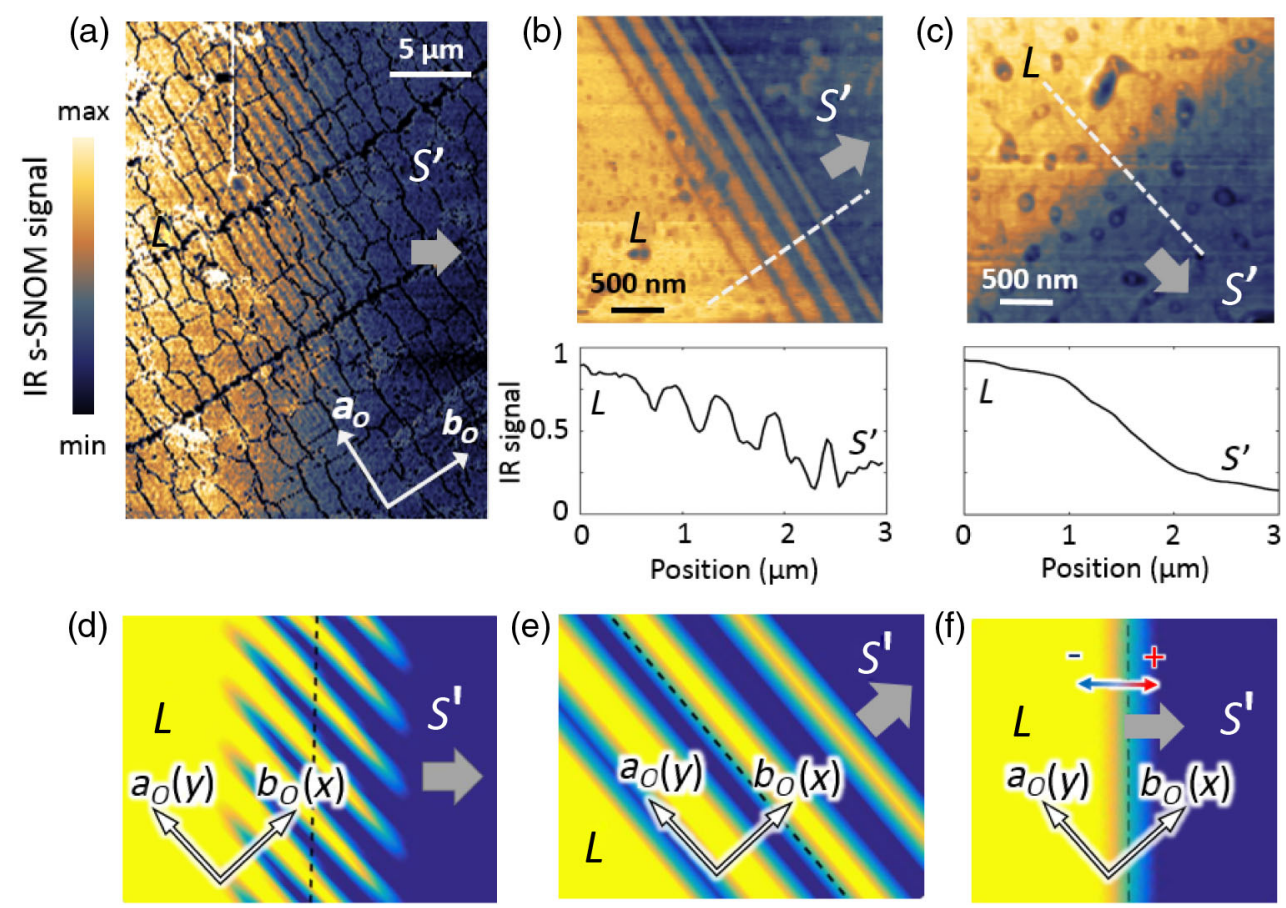

FIG. 3. Oriented stripe formation across the PB. (a)-(c) IR s-SNOM imaging (second harmonic) of $S^{\prime}-L$ phase boundaries on three different $\mathrm{Ca}_{2} \mathrm{RuO}_{4}$ samples. Lower panels in (b) and (c): Line cuts of the s-SNOM signal along the dashed lines in the upper panels. (d), (e) Results of the numerical minimization of the free energy for PB oriented at $45^{\circ}$ and $90^{\circ}$ to the crystalline $b_{o}$ direction [27]. Yellow indicates metal, and blue insulator. (f) shares the same parameter as (d) except using a larger driving force gradient $B$. The gray arrows in (a)-(f) indicate the direction of the $L$ state expansion (normal of the PB) under an increasing current. 
bright and dark stripes (lines of metallic and insulating behavior) is found to spontaneously develop within a few microns of the PB. The number and periodicity of these stripes vary in different regions. In some regions, no stripes at all are visible with only a simple steplike change in the optical response [Fig. 3(c)].

\section{ANALYSIS OF INSULATOR-METAL PHASE BOUNDARY}

\section{A. Minimal model for stripe formation}

We argue that the stripes arise from the difference in stress exerted by the electrons on the lattice in the two phases. While the insulating and metallic phases of $\mathrm{Ca}_{2} \mathrm{RuO}_{4}$ share the same Pbca symmetry, their lattice parameters are distinct. This difference leads to an elastic mismatch stress at insulator-metal phase boundaries. We focus here on the large in-plane orthorhombic anisotropy developed across the $\mathrm{Ca}_{2} \mathrm{RuO}_{4}$ structural transition $[21,23,42]$. The difference in the " $a_{o}$ " lattice parameter between the metallic $L$-Pbca phase and the $S^{\prime}$ state phase is within $0.03 \AA$, whereas the difference in the " $b_{o}$ " lattice parameters is $\sim 0.15 \AA$ [43], developing a spontaneous strain of approximately $3 \%$. We argue that the elastic energy cost of the $b_{o}$-axis misfit stress can be mitigated by a striped alternation of $L$ and $S^{\prime}$ structural phases parallel to the $a_{o}$ axis.

To analyze this effect theoretically, we consider a minimal model for an electronic IMT strongly coupled to strain, while including a heuristic driving force associated with the electrical current density. As explained in more detail below and in Supplemental Material [27], experimental and theoretical evidence suggests that, although a bulk metallic phase is sustained in the region near the negative electrode (far from the PB) [23,25], the metallic phase gradually reduces in depth closer to the $\mathrm{PB}$, tapering essentially into a thin layer at the top surface of the sample amid the striped coexistence region at the PB [Fig. 4(c)]. Thus, in our theoretical model, we assume that in the vicinity of the phase boundary there persists a thin metallic layer of depth $d$ much smaller than the sample thickness $D$ (Fig. S7 [27]). We express the spatially resolved free energy density associated with a first-order structure transition involving two locally stable phases: a metallic phase (order parameter $\phi=\phi_{M}$ ) and an insulating phase (order parameter $\phi=\phi_{I}$ ). Our free energy contains a driving term $f_{\text {current }}=f_{M}-f_{I}$, a difference in the energy density of the metallic and insulating phases, that increases linearly with the in-plane position $(\vec{r})$ along the direction $(\hat{n})$ of the current flow. This term is due physically to the spatial variation of the current density (as discussed in Sec. III in Supplemental Material [27]; the Peltier effect may also play a role). We write $f_{\text {current }}=A \hat{n} \cdot\left(\vec{r}-\vec{r}_{0}\right) \phi(\vec{r})$.

In the insulating phase near the transition the in-plane orthorhombic lattice parameters $a_{o}$ and $b_{o}$ are nearly equal, but in the metallic phase $b_{o}$ is longer by $2 \%$, implying that the IMT induces a stress, which we model as a misfit stress $\sigma_{x x}^{*}(r)=\sigma^{*}\left[\phi_{M}(r)-\phi_{I}\right]$ within the crystal, with the direction of stress $(x)$ aligned to the $b_{o}$ axis. The strain fields induced by this spontaneous stress extend throughout the crystal and are computed using the isotropic solid approximation of linear elasticity. As shown in Sec. III in Supplemental Material [27], after integration of the strain over the $z$ direction, the energy corresponding to the stress induced by a given spatial configuration of the order parameter may be expressed as an interaction between the domain walls (regions of high-order parameter gradient) separating metallic and insulating states [integration by parts from elastic energy $F_{\text {elastic }}$ in Eq. (S2) in Supplemental Material [27] ]:

$F_{\text {elastic }}^{\prime} \propto-\left(\sigma_{x x}^{*}\right)^{2} \int d x d y d x^{\prime} d y^{\prime} \frac{\partial \phi(x, y)}{\partial x} G_{x x}\left(\vec{r}, \vec{r}^{\prime}\right) \frac{\partial \phi\left(x^{\prime}, y^{\prime}\right)}{\partial x^{\prime}}$.

Here, $G$ is an elastic theory Green's function with appropriate boundary conditions enforcing net zero strain throughout the immobilized crystal, and the integral covers the crystal surface area spanning the $x$ and $y$ lateral directions. To complete the theory, we include the domain-wall energy density:

$$
f_{\mathrm{DW}} \propto \frac{1}{2} W|\nabla \phi(\vec{r})|^{2},
$$

where $W$ is the domain-wall energy parameter [27]. We see that $F_{\text {elastic }}^{\prime}$ comprises an attractive interaction between walls with the same "orientation" (the same sign of $[\partial \phi(x, y)] / \partial x$ ) and, if comparable to $f_{\mathrm{DW}}$, can induce spontaneous stripe formation.

\section{B. Emergence of surface stripes}

We minimize the resulting free energy numerically with respect to the spatial configuration $\phi(x, y)$ of the order parameter [27]; representative results are shown in Figs. 3(d)-3(f). We find that stripes can afford the minimal energy configuration of coexistent metal and insulator phases at the PB. This configuration particularly depends on the magnitude of the current density gradient, the relative values of the domain wall and elastic energies, and the orientation of the phase boundary with respect to the crystallographic axes (see Supplemental Material [27] for details of the calculation). We find that stripes are most easily produced when the PB normal coincides with the direction of spontaneous stress - that is, when the direction of the current is aligned to the in-plane $b_{o}(x)$ axis. Once formed, these stripes align perpendicular to the direction of the greatest elastic mismatch. The spatial extent of the striped region is set by the gradient of the current density change (denoted as $B$ in Supplemental Material [27]) across the PB from $L$ to the $S^{\prime}$ state, whereas the width of the stripes is set by the interplay of the domain wall and elastic energies. When $B$ is large enough, no stripes form 


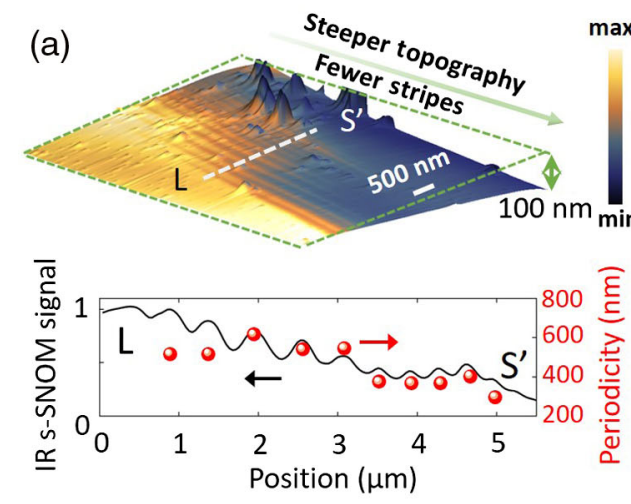

(c)

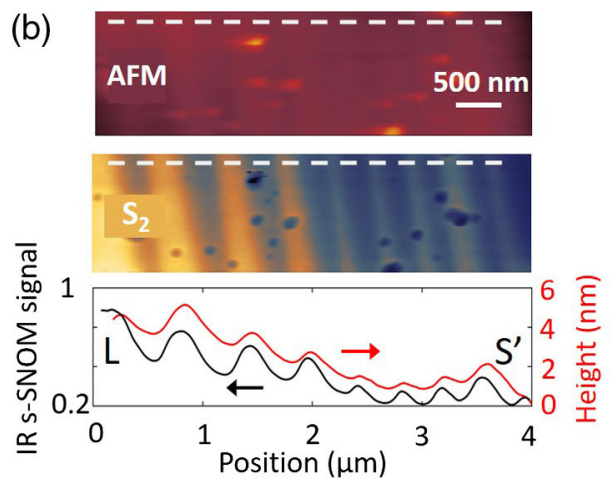

(d)

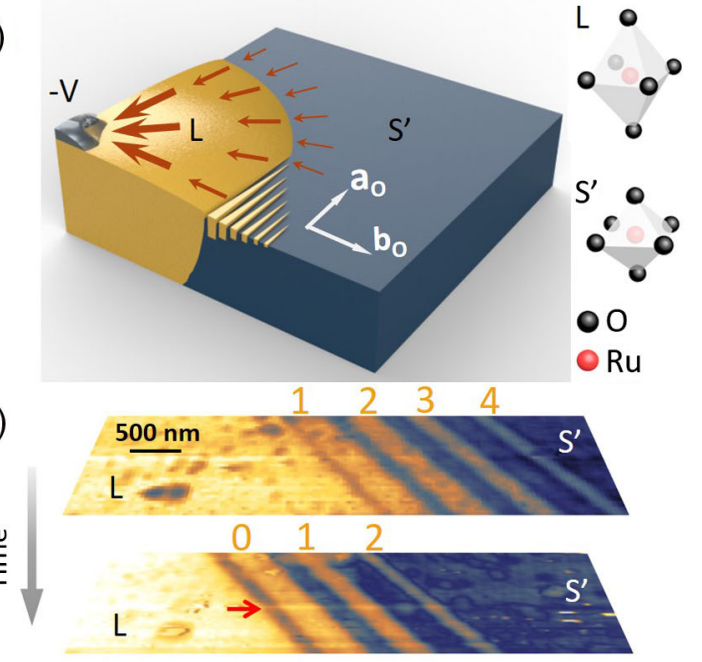

(e)

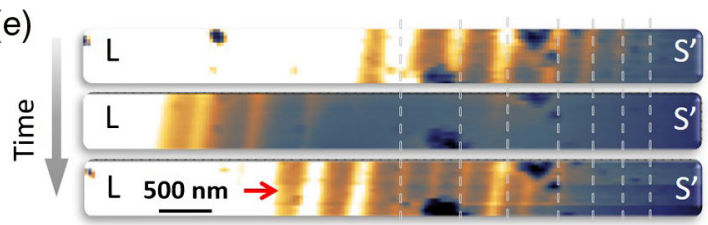

FIG. 4. Topography and progression or regression of the PB. (a) Top: The sample topography overlaid with the s-SNOM image in Fig. 2. For a larger height change from the $L$ to $S^{\prime}$ state, fewer stripes are observed. (b) AFM topography (top) and IR s-SNOM signal S2 imaging (middle) of eight stripes across the PB. The bottom figure shows that the variation in topography (red curve) has a one-to-one correspondence to the IR signal (black curve). (c) Schematic of a striped surface metallic layer at the PB, $L$ phase bulges due to a larger out-of-plane lattice constant; red arrows represent the magnitude and direction of the current density. (d), (e) Progression or regression of the PB taken with repeated IR s-SNOM imaging on three different samples under a current fluctuation around $12 \mathrm{~mA}$. (d) The occurrence of new metallic and insulating stripes with a width on the order of approximately 100-200 nm during the retraction of the phase front. (e) The fluctuation of the phase front. The current fluctuation and the time elapsed between the consecutive images taken in all the subfigures are less than $0.1 \mathrm{~mA}$ and $20 \mathrm{~min}$, respectively.

[comparison between Fig. 3(d) and 3(f)]. The relative widths of the insulating (or metallic) stripes shrink gradually towards the homogeneous phases [Fig. 3(e)], which coincides with our experimental observations [Fig. 3(b)]. The stripe periodicity is set by a combination of elastic energy, the gradient of the current density change, and the thickness $d$ of the metallic layer.

We next present results for the sample topography from atomic force microscopy, conducted concurrently with our IR nano-imaging. In Fig. 4(a), the s-SNOM image in Fig. 2 is overlaid with corresponding topography, demonstrating a gradual rise of the IR response (black curves) toward the higher end of the metallic side. Since the probing depth of s-SNOM is typically less than $50 \mathrm{~nm}[44,45]$, this gradual increase of the IR response suggests a growing depth of the metallic layer from the $S^{\prime}$ state to the $L$ phase across the PB, as schematically shown in Fig. 4(c). In addition, Fig. 4(b) reveals that the topography over the metallic stripes is approximately $1 \mathrm{~nm}$ higher than the insulating phase, which can be understood through the fact that the $S^{\prime}$-to- $L$ transition is accompanied by an out-of-plane lattice expansion by as much as $2 \%\left(c_{L} / c_{s}=1.023\right)$. Therefore, the surface metallic layer near the vicinity of the PB is estimated to be no more than a few hundred nanometers in depth. This estimate is much smaller than the full thickness of the crystal, which is hundreds of microns. These findings therefore justify the use of a thin metallic layer in our theoretical model. We also note that the alternative theoretical assumption of a domain wall extending approximately vertically over the whole thickness of the sample is not compatible with the spontaneous formation of stripes.

To conclude our findings, we demonstrate dynamic characteristics of the electrically induced PB. Figure 4(d) shows the retraction of the $L$ phase when the current is decreased by approximately $0.1 \mathrm{~mA}$ from $12.0 \mathrm{~mA}$. The top and bottom panels show the same sample region imaged by consecutive scans. Rather than a translational shift of preexisting stripe patterns, we observe that outer metallic stripes disappear ("3" and "4") or become narrower at their same fixed locations (" 1 " and " 2 "), whereas a new stripe (" 0 ") nucleates at locations prescribed by the stripe periodicity. In Fig. 4(e), multiple stripes are shown to emerge or 
vanish relative to the existing stripes. Such self-organized spontaneous phase separation mediated by long-range strain interactions remains an underexplored phenomenon in the context of the IMT in single crystals, motivating future study by nano-imaging probes with time-resolved capabilities.

\section{CONCLUSION}

In conclusion, we have used a combination of visiblefrequency microscopy and infrared nano-imaging to resolve the spatial structure and dynamics of the current-driven nonequilibrium IMT in $\mathrm{Ca}_{2} \mathrm{RuO}_{4}$ single crystals. The phase transition is initiated by a polarity-asymmetric nucleation of the current-induced bulk metallic phase at the negative electrode, consistent with strong electrostatic susceptibility due to electron-hole asymmetric electronic structure of the insulating $S$ phase. The phase nucleation proceeds by expansion of the metal phase across the sample as the current density is increased. A spontaneous surface stripe texture of coexisting metal and insulating phases is observed at the global metal-insulator phase boundary. We argue that this stripe formation is driven by the minimization of elastic mismatch strain and is well described by a minimal theory considering a thin surface metallic layer. Our results support an electrically induced insulator-metal transition scenario which is fundamentally distinct from the filamentary metallization common to high-field resistive switching reported in other oxides $[46,47]$. Future studies will include lowtemperature experiments of $\mathrm{Ca}_{2} \mathrm{RuO}_{4}$ single crystals and epitaxial thin films driven through the electrically induced IMT. Nano-imaging experiments under in situ applied strain would provide valuable insight to orbital ordering under static strain [48] to further clarify the microscopic mechanism of current-induced metallization in this compound.

\section{ACKNOWLEDGMENTS}

This work was supported by JSPS KAKENHI (No. JP15H05851, No. JP15H05852, No. JP15K21717, and No. JP17H06136) and the JSPS Core-to-Core program. C.S. acknowledges support of the JSPS International Research Fellowship (No. JP17F17027). A. S. M. and D. N. B. are supported by DOE-BES GrantNo. DE-SC0012375. D. N. B. is supported by the Gordon and Betty Moore Foundation's EPiQS Initiative through Grant No. GBMF4533. The authors gratefully acknowledge the efforts of M. Raschke and his group in developing the SINS instrument at the Advanced Light Source. The Advanced Light Source is supported by the Director, Office of Science, Office of Basic Energy Sciences, of the U.S. Department of Energy under Contract No. DE-AC02-05CH11231. G. L. C. acknowledges DOE DE-SC0012704. The work of A. J. M. and Q. H. was supported by the U.S. Department of Energy, Office of Science, Office of Basic Energy Sciences under Award No. DE-SC-0012375.
All authors contributed to the writing and editing of the manuscript.

The authors declare no competing financial interests.

[1] F. Chudnovskiy, S. Luryi, and B. Spivak, Switching Device Based on First-Order Metal-Insulator Transition Induced by External Electric Field, in Future Trends in Microelectronics: The Nano Millenium, edited by S. Luriy, J. M. Xu, A. Zaslavsky (John Wiley and Sons Ltd, New York, 2002), pp. $148-155$.

[2] C. Sow, S. Yonezawa, S. Kitamura, T. Oka, K. Kuroki, F. Nakamura, and Y. Maeno, Current-Induced Strong Diamagnetism in the Mott Insulator $\mathrm{Ca}_{2} \mathrm{RuO}_{4}$, Science 358, 1084 (2017).

[3] L. Cario, B. Corraze, A. Meerschaut, and O. Chauvet, Dielectric Breakdown and Current Switching Effect in the Incommensurate Layered Compound $(\mathrm{LaS})_{1.196} \mathrm{VS}_{2}$, Phys. Rev. B 73, 155116 (2006).

[4] S. Yamanouchi, Y. Taguchi, and Y. Tokura, Dielectric Breakdown of the Insulating Charge-Ordered State in $\mathrm{La}_{2-x} \mathrm{Sr}_{x} \mathrm{NiO}_{4}$, Phys. Rev. Lett. 83, 5555 (1999).

[5] C. Vaju, L. Cario, B. Corraze, E. Janod, V. Dubost, T. Cren, D. Roditchev, D. Braithwaite, and O. Chauvet, ElectricPulse-Driven Electronic Phase Separation, Insulator-Metal Transition, and Possible Superconductivity in a Mott Insulator, Adv. Mater. 20, 2760 (2008).

[6] V. Dubost, T. Cren, C. Vaju, L. Cario, B. Corraze, E. Janod, F. Debontridder, and D. Roditchev, Resistive Switching at the Nanoscale in the Mott Insulator Compound $\mathrm{GaTa}_{4} \mathrm{Se}_{8}$, Nano Lett. 13, 3648 (2013).

[7] B. Corraze et al. Electric Field Induced Avalanche Breakdown and Non-Volatile Resistive Switching in the Mott Insulators $\mathrm{AM}_{4} \mathrm{Q}_{8}$, Eur. Phys. J. Spec. Top. 222, 1046 (2013).

[8] V. Guiot, L. Cario, E. Janod, B. Corraze, V. T. Phuoc, M. Rozenberg, P. Stoliar, T. Cren, and D. Roditchev, Avalanche Breakdown in $\mathrm{GaTa}_{4} \mathrm{Se}_{8-x} \mathrm{Te}_{x}$ Narrow-Gap Mott Insulators, Nat. Commun. 4, 1722 (2013).

[9] Y. Taguchi, T. Matsumoto, and Y. Tokura, Dielectric Breakdown of One-Dimensional Mott Insulators $\mathrm{Sr}_{2} \mathrm{CuO}_{3}$ and $\mathrm{SrCuO}_{2}$, Phys. Rev. B 62, 7015 (2000).

[10] M. M. Qazilbash et al. Mott Transition in $\mathrm{VO}_{2}$ Revealed by Infrared Spectroscopy and Nano-Imaging, Science 318, 1750 (2007).

[11] A. S. McLeod et al. Nanotextured Phase Coexistence in the Correlated Insulator $\mathrm{V}_{2} \mathrm{O}_{3}$, Nat. Phys. 13, 80 (2016).

[12] J. Zhang, X. Tan, M. Liu, S. W. Teitelbaum, K. W. Post, F. Jin, K. A. Nelson, D. N. Basov, W. Wu, and R. D. Averitt, Cooperative Photoinduced Metastable Phase Control in Strained Manganite Films, Nat. Mater. 15, 956 (2016).

[13] M. H. Hamidian et al. Atomic-Scale Electronic Structure of the Cuprate d-Symmetry Form Factor Density Wave State, Nat. Phys. 12, 150 (2016).

[14] C. McGahan, S. Gamage, J. Liang, B. Cross, R. E. Marvel, R. F. Haglund, and Y. Abate, Geometric Constraints on Phase Coexistence in Vanadium Dioxide Single Crystals, Nanotechnology 28, 085701 (2017).

[15] J. Wu, Q. Gu, B. S. Guiton, N. P. De Leon, L. Ouyang, and H. Park, Strain-Induced Self Organization of Metal- 
Insulator Domains in Single-Crystalline $\mathrm{VO}_{2}$ Nanobeams, Nano Lett. 6, 2313 (2006).

[16] J. Cao et al. Extended Mapping and Exploration of the Vanadium Dioxide Stress-Temperature Phase Diagram, Nano Lett. 10, 2667 (2010).

[17] J. Trastoy, Y. Kalcheim, J. del Valle, I. Valmianski, and I. K. Schuller, Enhanced, Metal-Insulator Transition in $\mathrm{V}_{2} \mathrm{O}_{3}$ by Thermal Quenching after Growth, J. Mater. Sci. 53, 9131 (2018).

[18] S. Nakatsuji, S. I. Ikeda, and Y. Maeno, $\mathrm{Ca}_{2} \mathrm{RuO}_{4}:$ New Mott Insulators of Layered Ruthenate, J. Phys. Soc. Jpn. 66, 1868 (1997).

[19] C. Alexander, G. Cao, V. Dobrosavljevic, S. McCall, J. Crow, E. Lochner, and R. Guertin, Destruction of the Mott Insulating Ground State of $\mathrm{Ca}_{2} \mathrm{RuO}_{4}$ by a Structural Transition, Phys. Rev. B 60, R8422 (1999).

[20] F. Nakamura, T. Goko, M. Ito, T. Fujita, S. Nakatsuji, H. Fukazawa, Y. Maeno, P. Alireza, D. Forsythe, and S. R. Julian, From Mott Insulator to Ferromagnetic Metal: A Pressure Study of $\mathrm{Ca}_{2} \mathrm{RuO}_{4}$, Phys. Rev. B 65, 220402 (2002).

[21] P. Steffens et al. High-Pressure Diffraction Studies on $\mathrm{Ca}_{2} \mathrm{RuO}_{4}$, Phys. Rev. B 72, 094104 (2005).

[22] S. Riccò et al. In Situ Strain Tuning of the Metal-InsulatorTransition of $\mathrm{Ca}_{2} \mathrm{RuO}_{4}$ in Angle-Resolved Photoemission Experiments, Nat. Commun. 9, 4535 (2018).

[23] F. Nakamura, M. Sakaki, Y. Yamanaka, S. Tamaru, T. Suzuki, and Y. Maeno, Electric-Field-Induced Metal Maintained by Current of the Mott Insulator $\mathrm{Ca}_{2} \mathrm{RuO}_{4}$, Sci. Rep. 3, 2536 (2013).

[24] R. Okazaki, Y. Nishina, Y. Yasui, F. Nakamura, T. Suzuki, and I. Terasaki, Current-Induced Gap Suppression in the Mott Insulator $\mathrm{Ca}_{2} \mathrm{RuO}_{4}$, J. Phys. Soc. Jpn. 82, 103702 (2013).

[25] J. Bertinshaw et al. A Unique Crystal Structure of $\mathrm{Ca}_{2} \mathrm{RuO}_{4}$ in the Current Stabilized Metallic State, arXiv:1806.06455.

[26] H. A. Bechtel, E. A. Muller, R. L. Olmon, M. C. Martin, and M. B. Raschke, Ultrabroadband Infrared Nanospectroscopic Imaging, Proc. Natl. Acad. Sci. U.S.A. 111, 7191 (2014).

[27] See Supplemental Material at http://link.aps.org/ supplemental/10.1103/PhysRevX.9.011032 for details about sections. Section 1 introduces the experimental setup and measurement techniques. Section 2 gives more supporting evidence of the intermediate state $S$ ', discusses the role of thermal effects during the phase transition and shows an experiment of Peltier effect. Section 3 provides detail of the theoretical model used to reproduce the stripes at insulatormetal phase boundary.

[28] M. Liu et al. Phase Transition in Bulk Single Crystals and Thin Films of $\mathrm{VO}_{2}$ by Nanoscale Infrared Spectroscopy and Imaging, Phys. Rev. B 91, 245155 (2015).

[29] E. Dagotto, Complexity in Strongly Correlated Electronic Systems, Science. 309, 257 (2005).

[30] M. Liu, A.J. Sternbach, and D. N. Basov, Nanoscale Electrodynamics of Strongly Correlated Quantum Materials, Rep. Prog. Phys. 80, 014501 (2017).

[31] R. Kumai, Current-Induced Insulator-Metal Transition and Pattern Formation in an Organic Charge-Transfer Complex, Science 284, 1645 (1999).

[32] A. Pustogow, A. S. McLeod, Y. Saito, D. N. Basov, and M. Dressel, Internal Strain Tunes Electronic Correlations on the Nanoscale, Sci. Adv. 4, eaau9123 (2018).
[33] M. Braden, G. André, S. Nakatsuji, and Y. Maeno, Crystal and Magnetic Structure of $\mathrm{Ca}_{2} \mathrm{RuO}_{4}$; Magnetoelastic Coupling and the Metal-Insulator Transition, Phys. Rev. B 58, 847 (1998).

[34] M. Sakaki, N. Nakajima, F. Nakamura, Y. Tezuka, and T. Suzuki, Electric-Field-Induced Insulator-Metal Transition in $\mathrm{Ca}_{2} \mathrm{RuO}_{4}$ Probed by X-Ray Absorption and Emission Spectroscopy, J. Phys. Soc. Jpn. 82, 093707 (2013).

[35] Q. Han and A. Millis, Lattice Energetics and CorrelationDriven Metal-Insulator Transitions: The Case of $\mathrm{Ca}_{2} \mathrm{RuO}_{4}$, Phys. Rev. Lett. 121, 067601 (2018).

[36] T. Mizokawa, L. H. Tjeng, G. a. Sawatzky, G. Ghiringhelli, O. Tjernberg, N. B. Brookes, H. Fukazawa, S. Nakatsuji, and Y. Maeno, Spin-Orbit Coupling in the Mott Insulator $\mathrm{Ca}_{2} \mathrm{RuO}_{4}$, Phys. Rev. Lett. 87, 077202 (2001).

[37] E. Gorelov, M. Karolak, T. O. Wehling, F. Lechermann, A. I. Lichtenstein, and E. Pavarini, Nature of the Mott Transition in $\mathrm{Ca}_{2} \mathrm{RuO}_{4}$, Phys. Rev. Lett. 104, 226401 (2010).

[38] C. G. Fatuzzo et al. Spin-Orbit-Induced Orbital Excitations in $\mathrm{Sr}_{2} \mathrm{RuO}_{4}$ and $\mathrm{Ca}_{2} \mathrm{RuO}_{4}:$ A Resonant Inelastic X-Ray Scattering Study, Phys. Rev. B 91, 155104 (2015).

[39] Ismail, J. Zhang, R. Matzdorf, T. Kimura, Y. Tokura, and E. Plummer, Surface Lattice Dynamics of Layered Transition Metal Oxides: $\mathrm{Sr}_{2} \mathrm{RuO}_{4}$ and $\mathrm{La}_{0.5} \mathrm{Sr}_{1.5} \mathrm{MnO}_{4}$, Phys. Rev. B 67, 035407 (2003).

[40] R. G. Moore, J. Zhang, V. B. Nascimento, R. Jin, J. Guo, G. T. Wang, Z. Fang, D. Mandrus, and E. W. Plummer, A Surface-Tailored, Purely Electronic, Mott Metal-to-Insulator Transition, Science 318, 615 (2007).

[41] J.S. Lee et al. Bond-Length Dependence of ChargeTransfer Excitations and Stretch Phonon Modes in Perovskite Ruthenates: Evidence of Strong p-d Hybridization Effects, Phys. Rev. B 70, 085103 (2004).

[42] Y. F. Gao, W. Lu, and Z. Suo, A Mesophase Transition in a Binary Monolayer on a Solid Surface, Acta Mater. 50, 2297 (2002).

[43] O. Friedt, M. Braden, G. Andre, P. Adelmann, S. Nakatsuji, and Y. Maeno, Structural and Magnetic Aspects of the Metal Insulator Transition in $\mathrm{Ca}_{2-x} \mathrm{Sr}_{x} \mathrm{RuO}_{4}$, Phys. Rev. B 63, 174432 (2001).

[44] L. M. Zhang, G. O. Andreev, Z. Fei, A. S. McLeod, G. Dominguez, M. Thiemens, A. H. Castro-Neto, D. N. Basov, and M. M. Fogler, Near-Field Spectroscopy of Silicon Dioxide Thin Films, Phys. Rev. B 85, 075419 (2012).

[45] A. A. Govyadinov, S. Mastel, F. Golmar, A. Chuvilin, P. S. Carney, and R. Hillenbrand, Recovery of Permittivity and Depth from Near-Field Data as a Step toward Infrared Nanotomography, ACS Nano 8, 6911 (2014).

[46] D.-H. Kwon et al. Atomic Structure of Conducting Nanofilaments in $\mathrm{TiO}_{2}$ Resistive Switching Memory, Nat. Nanotechnol. 5, 148 (2010).

[47] M.-J. Lee et al. A Fast, High-Endurance and Scalable Non-Volatile Memory Device Made from Asymmetric $\mathrm{Ta}_{2} \mathrm{O}_{5-x} / \mathrm{TaO}_{2-x}$ Bilayer Structures, Nat. Mater. 10, 625 (2011).

[48] L. Miao, W. Zhang, P. Silwal, X. Zhou, I. Stern, T. Liu, J. Peng, J. Hu, D. H. Kim, and Z. Q. Mao, Epitaxial Strain Effect on Transport Properties in $\mathrm{Ca}_{2-x} \mathrm{Sr}_{x} \mathrm{RuO}_{4}$ Thin Films, Phys. Rev. B 88, 115102 (2013). 